\title{
INVESTIGACIONES
}





\title{
La regla de precedente
}

\author{
Fabio Enrique Pulido Ortiz*
}

\begin{abstract}
RESUMEN
En este artículo se explican las características de la regla de precedente (RP). La RP, en sentido amplio, es aquella regla de los ordenamientos jurídicos que configura el funcionamiento de los precedentes judiciales $(P J)$. En los ordenamientos jurídicos puede existir de forma contingente una $R P$ que establezca el carácter vinculante u obligatorio de los PJ. En este caso la RP desarrolla, por lo menos, tres funciones: 1) establecer las condiciones para la identificación de los PJ, 2) fijar las competencias para crear, derogar y aplicar los PJ vinculantes y 3) consagrar la forma en que los PJ exigen (obligan) la realización de actos o acciones. A partir de la distinción entre vinculación y obligación, se subraya que las sanciones son las consecuencias contingentes del incumplimiento del deber de seguir la RP y que la nulidad (o invalidez) es la consecuencia necesaria del incumplimiento de las condiciones vinculantes definidas en la RP.
\end{abstract}

Precedentes judiciales; precedentes vinculantes; precedentes obligatorios; teoría del derecho

\section{The rule of precedent}

\section{Abstract}

This paper explains the main features of the rule of precedent $(R P)$. The RP -in lato sensu-is the rule that defines the legal operation of judicial precedents (PJ). It is possible that each legal

* Abogado, Universidad Católica de Colombia. Magíster en Derecho Constitucional, Universidad de Palermo, Argentina. Doctor en Derecho, Universidad de Buenos Aires, Argentina. Profesor Asociado de la Facultad de Derecho y Ciencias Políticas de la Universidad de La Sabana, Colombia. Correo electrónico: fabio.pulido@unisabana.edu.co. ORCID: https://orcid.org/0000-0002-1100-9962.

Este artículo forma parte de los resultados del proyecto de investigación "la política con toga: un estudio del activismo judicial” (DER-60-2019) financiado por la Dirección General de Investigaciones de la Universidad de La Sabana.

Una versión preliminar de las ideas plasmadas en este texto fueron presentadas y discutidas en el seminario permanente acerca de precedente judicial organizado por el Centro de Estudios Constitucionales de la Suprema Corte de Justicia de la Nación-México. Agradezco las observaciones de Alejandra Martínez Verástegui (CEC SCJN), Ana María Ibarra Olguín (CEC SCJN) y Rodrigo Camarena González (ITAM). Agradezco asimismo a Carlos Bernal Pulido, Pablo Rivas Robledo, Sergio Morales Barreto, Raquel Sarria, Jacobo Gómez y Gabriela González quienes, en las discusiones del grupo de investigación Justicia, Ámbito Público y Derechos Humanos de la Universidad de La Sabana, comentaron y criticaron versiones previas del artículo. Por último, los evaluadores anónimos del artículo ayudaron significativamente a mejorar la calidad del texto con sus observaciones.

Artículo recibido el 23.7.2020 y aceptado para su publicación el 28.4.2021. 
order contains a contingent RP in stricto sensu to define the conditions of binding and mandatory PJ. About this matter, the RP carries out three principal functions: 1) to establish the conditions to identify PJ; 2) to fix the legal powers for create, overrule and apply the PJ, and 3) to define the way in which the PJ commands actions and the consequences of non-compliance. Based on distinction between binding and obligation, it is highlighted that sanctions are the contingent consequences of disobey RP and that nullity (or invalidity) is the necessary consequence of the non-compliance of the binding conditions defined by the RP.

Judicial precedents; binding precedents; mandatory precedents; legal theory

\section{INTRODUCCIÓN}

$\mathrm{E}$ n los ordenamientos jurídicos actuales los precedentes judiciales (en adelante PJ) tienen una relevancia indudable. Con independencia de la tradición jurídica, es posible advertir distintas formas de regla de precedente (en adelante RP) ${ }^{1}$. Al respecto existe una distinción básica entre la RP y los PJ. La RP se refiere a la regla que define el funcionamiento normativo de los PJ. Los PJ son normas generales y abstractas originadas en una providencia judicial ${ }^{2}$.

A manera de ejemplo, supongamos que un tribunal constitucional establece (en su ratio decidendi) que "las empresas de salud tienen a su cargo el suministro de pañales a los adultos mayores con incontinencia”. Asumamos que en el ordenamiento jurídico se tiene definido que la ratio decidendi de esas sentencias vinculan a los jueces en casos posteriores. Con base en este ejemplo puede verse la diferencia entre la regla que establece las prestaciones a cargo de las empresas de salud (esto el PJ) y la regla que define que los PJ son vinculantes (esto es la RP). En desarrollo de esta distinción, el objetivo de este artículo es explicar las características de la regla de precedente (RP). El propósito no es describir cómo -en las prácticas jurídicas vigentes- se implementa esta regla, sino plantear un modelo conceptual que sirva de referente para esa tarea.

Se argumenta que la RP en sentido amplio (aquella regla del ordenamiento jurídico que configura el funcionamiento de los PJ) es una regla necesaria de los ordenamientos jurídicos. No obstante, puede existir de forma contingente una RP que establezca el carácter vinculante u obligatorio de los PJ. En este caso la RP desarrolla, por lo menos, tres funciones: 1) establecer las condiciones para la identificación de los PJ; 2) fijar las competencias para la creación y aplicación de los PJ vinculantes; y 3) consagrar la forma en que los PJ exigen (obligan) la realización de actos o acciones. Por último, y a partir de la distinción entre vinculación y obligación, se subraya que las sanciones son las consecuencias contingentes por desobedecer la RP, mientras que la nulidad (o invalidez) es la consecuencia necesaria del incumplimiento de la RP vinculante.

${ }^{1}$ Véase, entre otros, Komarek, 2013, pp. 103-140, 149-171; Bravo-Hurtado, 2013, pp. 549 - 576 y Bernal Pulido, C., 2009, pp. 153-158.

${ }^{2}$ Núñez Vaquero, 2016, p. 134 y Schauer, 2013, p. 56. 


\section{LA NECESIDAD DE LA REGLA DE PRECEDENTE}

La RP en general se refiere a la regla del ordenamiento jurídico que configura el funcionamiento de los PJ. La RP, por esta razón, alude a la forma en que se puede implementar la RP en cada ordenamiento jurídico. Así, por consiguiente, esta regla puede adoptar tres formas: 1) la RP vinculante/obligatoria, 2) la RP como autorización y 3) la RP como rechazo de los $\mathrm{PJ}^{3}$.

La primera forma de RP es aquella que establece que los PJ vinculan u obligan ${ }^{4}$. La RP es vinculante en el sentido que se constituye en una condición necesaria para la validez de los actos de los operadores jurídicos. Asimismo, la RP es obligatoria cuando exige (i.e. prescribe) la aplicación de los PJ. La segunda forma es la RP como autorización. En este sentido, la RP autoriza a los operadores jurídicos el empleo de los PJ en la justificación de sus actos (sin vincular, es decir, sin condicionar la validez de los actos al cumplimento de los PJ) y permite que los operadores jurídicos resuelvan conforme a los PJ (i.e. sin obligar ni prohibir la aplicación del PJ). ${ }^{5}$ La tercera forma de RP es aquella que rechaza el uso de los PJ. Así, en un ordenamiento jurídico la RP puede establecer que el uso de los PJ afecta la validez de los actos jurídicos $(v \cdot g r$. si una sentencia se fundamenta en el PJ "X" entonces es inválida). Incluso, puede ser el caso que la RP prohíba el uso o seguimiento de $\mathrm{PJ}^{6}$.

Teniendo en cuenta lo anterior, la RP en sentido amplio es necesaria, esto es, todos los ordenamientos jurídicos tienen una regla acerca de los PJ. En cambio, la RP en sentido estricto es contingente porque en cada ordenamiento jurídico adopta distintas formas de RP e incluso puede implementar varias de ellas dentro del mismo. Conceptualmente existen solo dos posibilidades: por un lado, los ordenamientos jurídicos (por medio de su RP) pueden aceptar el uso y seguimiento de los PJ. En este escenario, la RP puede ser 1) vinculante u obligatoria o 2) una autorización (sin vincular ni obligar). Por otro lado, la RP puede rechazar el uso y seguimiento de PJ. En otras palabras, todo ordenamiento jurídico adopta alguna forma de RP: vinculando, obligando, autorizando o rechazando.

La RP en estricto sentido se refiere a la configuración específica de cada una de las formas de RP en los ordenamientos jurídicos y esta configuración es contingente a cada uno de ellos. En otros términos, todos los ordenamientos jurídicos configuran alguna forma de RP, pero existen diversas formas de hacerlo. Unos contienen una RP que exige la aplicación del PJ, otros pueden autorizar (sin exigir) y otros pueden, incluso,

${ }^{3}$ Suele reducirse este tema a dos tipos de RP: persuasivo y una autoritativo. Véase, BronAUGH, 1987 , pp. 216-247. Dobbins, 2010 pp. 1453-1496.

${ }^{4}$ Estas diferencias han sido descuidadas en la mayoría de trabajos referentes al tema. Véase Cross \& Harris, 2012, pp. 123-152; PeCZenik, 1997 pp. 461-479 y Duxbury, 2008, pp. 12-22. La importancia de diferenciar esas variables, y el carácter central de la vinculación en la explicación de la RP, ha sido recientemente planteada por ArRiagada, 2021, pp. 361-396 y NúÑEZ VAquero, 2021, pp. 329-360.

${ }^{5}$ El ejemplo más conocido de este tipo de RP es el PJ persuasivo. Véase DobBins, 2010, pp. 14621463 y Bronaugh, 1987, pp. 216-247.

${ }^{6}$ Cross y Harris ejemplifican esta regla en el derecho francés que prohíbe que los jueces dicten reglas generales y, en consecuencia, que sigan esas reglas de otros jueces (Cross \& HARris, 2012, p. 36.) 
prohibirlo. Es más, dentro de un mismo ordenamiento jurídico se pueden configurar o implementar distintas formas o tipos de $\mathrm{RP}^{7}$.

En este punto es indispensable subrayar un presupuesto conceptual. Una característica de los ordenamientos jurídicos contemporáneos es que los poderes o potestades de sus autoridades están definidas (o constituidas) en reglas jurídicas (reglas de competencia) que configuran y hacen posible el ejercicio válido de esos poderes. Como veremos más adelante, la RP es una regla de segundo orden en la que se definen, justamente, las condiciones para la identificación, creación y aplicación válida de $\mathrm{PJ}^{8}$. Es admisible resaltar que este tipo de poderes dependen para su ejercicio válido de una norma que los haga posibles, esto quiere decir que, en caso de que no exista una norma acerca de la creación o aplicación del PJ, entonces debe entenderse que el ordenamiento jurídico contiene una RP de rechazo de los PJ. Podría discutirse que estas condiciones para el ejercicio de poderes de las autoridades son accidentales y que, por tanto, la RP en sentido amplio es contingente en el entendido que puede ser el caso en el que no existan normas que nieguen, autoricen, ni exijan el uso del PJ. Pero para sostener esta idea, tendría que negarse que un elemento necesario para la explicación de los poderes jurídicos es la existencia de reglas de competencia. Estas reglas posibilitan el ejercicio de los poderes jurídicos (como el de crear o aplicar el PJ). En otras palabras, no es posible el ejercicio de un poder jurídico sin la existencia de una norma de competencia que lo constituya.

Además, en la explicación del ejercicio de los poderes públicos en los ordenamientos jurídicos contemporáneos es central el principio de legalidad, esto es el principio de acuerdo con el que son válidos los actos de los poderes públicos que sean efectuados conforme a las reglas de competencia (y, en consecuencia, que son inválidos los actos de los poderes públicos realizados por fuera o en contra de esas normas) ${ }^{9}$. A causa de la centralidad de este principio en las prácticas jurídicas, la explicación de las condiciones de validez del ejercicio de los poderes públicos (por lo menos en el contexto de un estado de derecho) depende de las normas que hacen posibles estos poderes ${ }^{10}$. El principio de legalidad, en definitiva, establece una clausura en el sistema de definición de los poderes públicos, de forma tal que solo es válido el ejercicio de aquellos poderes contemplados en las reglas de competencia. Por razones de espacio, no es posible desarrollar con mayor detalle esta discusión. Basta para los propósitos de este trabajo subrayar que, bien sea por las razones conceptuales asociadas a la noción de reglas de competencia o por la

${ }^{7}$ Cross \& Harris, 2012, pp. 61-106; Duxbury, 2008, 67 -73; Marshall, 1997, p. 505.

${ }^{8} \mathrm{La}$ RP es una regla que contiene varias reglas (competencias, definiciones e incluso prescripciones) sobre PJ. Por ello, es una regla secundaria en dos sentidos: 1) está conformada por normas constitutivas en el ordenamiento jurídico (i.e. constituye las formas válidas de crear, identificar, aplicar los PJ); y 2) está conformada por metanormas, esto es, reglas sobre reglas. En este segundo sentido, la RP puede -contingentemente- ser prescriptiva. Aun cuando es cierto que la RP contiene reglas constitutivas, no todas esas reglas constitutivas son de competencia porque algunas son reglas definicionales $(v . g r$. definiciones sobre la distinción entre ratio decidendi y obiter dicta). Además, la RP puede contener prescripciones: en algunos ordenamientos jurídicos, por ejemplo, los jueces deben aplicar los PJ sometidos incluso a sanciones disciplinarias y penales.

${ }^{9}$ Guastini, 2001, p. 123.

${ }^{10}$ Véase Waldron 2020, en especial las secciones 5 y 9. 
centralidad del principio de legalidad, lo cierto es que, en aquellos ordenamientos jurídicos que no contemplen las condiciones para crear, modificar y aplicar PJ, se debe entender que existe implícitamente una RP de rechazo.

Teniendo en cuenta estas precisiones, el siguiente aparatado se enfocará en el análisis de la RP vinculante.

\section{LA REGLA DE PRECEDENTE COMO REGLA DE SEGUNDO ORDEN}

Los ordenamientos jurídicos están conformados por dos tipos de reglas: las reglas primarias (o de primer orden) y las reglas secundarias (o de segundo orden) ${ }^{11}$. Las reglas primarias son aquellas que se encargan de dirigir la acción exigiendo o permitiendo la realización (o no realización) de determinados comportamientos. Las reglas secundarias son reglas sobre reglas (normas acerca de cómo identificar, crear o aplicar reglas). Existe, no obstante, una ambigüedad en el concepto de reglas secundarias. Por un lado, con el concepto de reglas secundarias se puede hacer referencia a las normas de los ordenamientos jurídicos cuya finalidad es definir cómo identificar, crear, modificar, derogar o aplicar otras reglas. De acuerdo con esto, la RP es una regla secundaria que define el funcionamiento normativo de los PJ. Por otro lado, el concepto de reglas secundarias puede emplearse para designar las normas cuyo objeto es otra norma. En este segundo sentido, las reglas secundarias pueden definir actos normativos $(v$ gr. la creación de reglas) pero también prescribir acciones (exigir la aplicación de reglas). En conclusión, la RP es una regla de segundo orden, no solo en el primer sentido (esto es como una norma que define las condiciones para la identificación, creación y aplicación válida de PJ), sino también puede ser una regla secundaria en el segundo sentido, por ejemplo en aquellos ordenamientos jurídicos que, además de establecer una RP vinculante, se establece el deber de aplicar PJ (i.e. la RP como obligación).

En tanto regla de segundo orden (en el primero de los sentidos) la RP establece las condiciones en que los PJ son vinculantes. En esta tarea, la RP define: a) los criterios para la identificación de los PJ, b) las competencias para creación y aplicación de los $\mathrm{PJ}$; y c) los criterios para establecer el significado de los $\mathrm{PJ}^{12}$.

${ }^{11}$ Las primeras fundamentaciones filosóficas de la distinción de niveles o tipos de reglas puede verse en HART, 1994 y VON WRIGHT, 1963. Las críticas más notables acerca del asunto se dirigen a caracterizaciones específicas de la misma, pero no se discute la distinción en sí misma. Véase, por ejemplo, RAZ, J. 1979, pp. 92-93 y COLEMAN, pp. 84-86.

${ }^{12}$ Con diferencias, la tesis de que la RP es una regla secundaria está formulada en Pulido 2020 y Núñez 2020. Para el primero, la RP es un tipo de regla de adjudicación y de cambio. Para el segundo, la RP “implica al menos tres normas: una primera norma acerca de la competencia para dictar precedentes; una segunda que condiciona la regularidad de la decisión jurisdiccional y la aplicabilidad de la norma individual contenida en el decisum de aquella; y una tercera (contingente) sobre la competencia para el seguimiento de precedentes" (p. 330). 


\section{a. La identificación de los precedentes judiciales}

La existencia de una RP vinculante supone la existencia de decisiones judiciales que contienen normas generales y abstractas que constituyen a los $\mathrm{PJ}^{13}$. Las normas jurídicas correlacionan un caso (conjunto de propiedades que caracterizan un tipo de acción o acto) y una solución normativa (prohibición, obligación, facultad, potestad) ${ }^{14}$. Son generales cuando sus destinatarios están constituidos por una clase de personas, es decir, que se dirigen a una categoría de personas (niños, adultos, extranjeros, jueces etc.). Asimismo, son abstractas cuando el caso está conformado por hechos hipotéticos, esto es por tipos o clases de acciones o actos ( $v$ gr. usar cinturón de seguridad, votar o legislar). Por tanto, las normas jurídicas generales y abstractas (como las normas constitucionales, las de un código o, en nuestro caso, un PJ) son aquellas en las que sus destinatarios son una clase de personas y el caso es hipotético ${ }^{15}$.

La RP define las normas jurídicas generales y abstractas que constituyen los PJ. Existen, al respecto, dos variables: 1) la determinación de las providencias judiciales que constituyen a los PJ y 2) la definición del contenido normativo que -en específico- constituye al PJ. En relación con la primera variable, la RP puede establecer - por ejemplo- que solo las sentencias generan los PJ (es decir, excluyendo otro tipo de providencias). Puede también delimitar que solo las sentencias de determinados órganos judiciales ( $v, g r$. los órganos de cierre) son constitutivos del PJ.

La segunda variable es más compleja. Esta variable depende, además, de la identificación de los poderes normativos de los jueces. Los poderes normativos se refieren a la capacidad que tienen las autoridades de afectar las acciones y actos de otros individuos (o, incluso, de ellos mismos) ${ }^{16}$. Son poderes normativos en el sentido que tienen la capacidad de modificar los deberes de los individuos o condicionar la validez de sus $\operatorname{actos}^{17}$. Un Juez tiene un poder normativo respecto de un individuo si es el caso que sus sentencias tienen la capacidad de obligar a ese individuo a realizar (o no realizar) una acción ( $v . g r$. entregar una suma de dinero) o de afectar la validez de los actos de ese individuo ( $v, g r$. anular un contrato).

Como se dijo, los PJ son normas generales y abstractas, por ello, en el contexto de la RP, los jueces ejercen poderes normativos que tienen efectos generales y abstractos. Al definir cuáles de esas normas (i.e. de las normas generales y abstractas creadas por los jueces) son vinculantes, la dogmática jurídica suele recurrir al concepto de ratio decidendi,

13 Alexander \& Sherwin, 2007, pp. 27-50; Núñez Vaquero, 2016, p. 134 y Schauer, 2013, p. 56. Es conceptualmente posible la existencia de RP y que no existan PJ (esto es que no se haya creado ningún precedente). Pero lo importante no es esta eventualidad, sino que las providencias tengan la "capacidad" de originar esas normas, aun cuando en efecto no existan PJ. Una capacidad se refiere a la potencia de un sujeto u objeto de ser o hacer algo. Acerca de esta noción véase RAZ, 2019, p.- 1-17.

14 Alchourrón y Bulygin, 1987, p. 38.

15 Guastini, 2009, p. 95. Hernández, 2002, p. 221.

${ }^{16}$ Raz, 2019, pp. 1-17; Raz, 1990, pp. 98-106.

${ }^{17}$ RAZ, 2019, pp. 1-17. 
esto es, la regla que constituye la base de la decisión judicial y que corresponde al fundamento normativo directo para la resolución del problema jurídico a la luz del decisum de la providencia ${ }^{18}$. La noción de ratio decidendi-como se sabe-está asociada al principio de razón suficiente de las decisiones judiciales, esto es que toda providencia judicial debe fundarse en una norma general y abstracta que justifica la decisión establecida en el fallo. La ratio decidendi está constituida por la norma general y abstracta que sirve de fundamento a la resolución del problema jurídico y que, se supone, guarda unidad de sentido son el dispositivo del fallo ${ }^{19}$.

No obstante, reducir los PJ a la noción de ratio decidendi deja por fuera otros poderes normativos que son ejercidos por los jueces en las prácticas jurídicas actuales y que también son constitutivos de normas generales y abstractas. Recordemos algo básico: una sentencia judicial es un acto normativo que resuelve autoritativamente (i.e. de forma obligatoria o vinculante) y con valor de cosa juzgada (i.e. con carácter definitivo) un problema jurídico (i.e. un problema acerca de la adecuada aplicación del derecho a una situación definida $)^{20}$. En los ordenamientos jurídicos que reconocen la $\mathrm{RP}$ vinculante, los jueces - por medio de sus providencias judiciales- ejercen, al menos, dos poderes normativos: 1) resolver de forma autoritativa los problemas jurídicos que les son planteados y 2) establecer contenidos normativos que regulan casos más allá de lo decidido en el fallo respectivo. La cosa juzgada, esto es, la fuerza normativa de la decisión judicial respecto de los problemas específicos planteados a los jueces, se refiere a la primera función. Los jueces -en virtud del principio de cosa juzgada-tienen el poder normativo de determinar autoritativamente los derechos y deberes de otras personas e instituciones ${ }^{21}$. Los PJ del segundo tipo de poderes, es decir, el de originar normas jurídicas generales y abstractas ${ }^{22}$.

La diferencia entre la cosa juzgada y el PJ radica, en principio, en que el poder normativo ejercido mediante el PJ trasciende, de forma necesaria, la resolución de la litis, y se constituye en regla para la decisión en casos futuros, mientras que los efectos de la cosa juzgada se limitan a la resolución de un asunto concreto. No obstante, se debe precisar que en los ordenamientos jurídicos contemporáneos existen tribunales que tienen el poder de resolver problemas abstractos (por ejemplo, la definición en abstracto de la constitucionalidad de normas), y al mismo tiempo las decisiones de dichos tribunales tienen efectos erga omnes. Así, por ejemplo, si un tribunal constitucional considera que

${ }^{18}$ Cross \& Harris, 2012, pp. 61-106; Marshall, 1997, pp. 503-517; López, 2006, pp. 217-260 y GARAY, 2013, pp. 113-141.

19 Existe una amplia discusión -incluso en el common law- del concepto de ratio decidendi. Véase Duxbury, N. 2008, pp. 67-92; Chiassoni, 2015, pp. 31-42; Núñez Vaquero, 2016, pp. 130-135; Twining y Miers, 2010, pp. 304-312; y Komarek, 2013, pp. 149-171.

${ }^{20}$ Hernández, 2008, pp. 15-20.

${ }^{21}$ Moreso y Queralt, 2014, p. 154; y Hart, 1994, p. 141-147.

${ }^{22}$ Alexander \& Sherwin, 2007, pp. 27-50 
la norma $\mathrm{X}$ es inconstitucional entonces tiene el poder de expulsarla del ordenamiento jurídico con efectos generales y abstractos ${ }^{23}$.

Además, los tribunales constitucionales han desarrollado la práctica de condicionar las sentencias de constitucionalidad abstracta. Por lo general, se espera que el fallo de estas sentencias establezca si una norma jurídica es o no constitucional. Sin embargo, en determinadas circunstancias, los tribunales constitucionales ofrecen respuestas "condicionadas"; es decir, si se plantea que la norma jurídica X es inconstitucional por violar la norma constitucional $\mathrm{Y}$, el tribunal podría responder que $\mathrm{X}$ es constitucional si, $\mathrm{y}$ solo si, se entiende que debe contemplar el contenido normativo P. En estos escenarios lo que suele ocurrir es que el tribunal constitucional, o delimita la norma a un sentido específico o incluye supuestos normativos adicionales. Y en cualquiera de los dos casos esos contenidos son normas generales y abstractas establecidas en una providencia judicial ${ }^{24}$.

Por último, algunos tribunales profieren órdenes generales como mecanismo para remediar las situaciones que les son sometidas a su decisión. En general, los jueces resuelven los problemas jurídicos mediante decisiones con efectos que vinculan solo a las partes del proceso (efectos interpartes). Una característica del decisum de estas providencias es que la norma allí identificada vincula, generalmente, solo a las partes del proceso $^{25}$. Sin embargo, existen situaciones en las que los jueces (principalmente los jueces constitucionales) argumentan que, a pesar de que el asunto sea de aquellos que exigen una decisión interpartes, diferentes factores -como la inactividad de los otros poderes públicos- justifican que el tribunal respectivo realice órdenes generales que en muchos casos vinculan a la administración e incluso al órgano legislativo ${ }^{26}$.

Cuando los jueces tienen el poder de crear normas jurídicas generales y abstractas en el decisum de sus providencias, es posible diferenciar entre el PJ en estricto sentido y el PJ en amplio sentido. En el primero, el PJ se refiere a la ratio decidendi de las providencias judiciales y que en virtud de la RP puede ser vinculante. En el segundo sentido, el PJ se refiere a todas las normas generales y abstractas originadas en una providencia judicial, lo que incluye -además de la ratio decidendi-a las normas generales y abstractas que son creadas en los decisum de las providencias ${ }^{27}$. La explicación de la RP, por tanto, debe dar cuenta de los diferentes poderes normativos de los jueces de crear contenidos generales y abstractos. Estos poderes, como se dijo, en muchos de los ordenamientos jurídicos actuales van más allá de la noción de ratio decidendi ${ }^{28}$.

${ }^{23}$ ZHOU, 2019, p. 277-299.

24 Díaz Revorio, 2000, pp. 1799-1824

${ }^{25}$ ZHOU, 2019, p. 277-299. Algunos ordenamientos jurídicos han incluido, además, los efectos que el decisum vincula a quienes fueron parte del proceso y a quienes fueron parte del problema jurídico que originó la sentencia, pero no hicieron parte del proceso. Este es el caso de los efectos en amparos colectivos y acciones de clase. Véase Moreno Cruz, 2017, pp. 61-119.

${ }^{26}$ Estas órdenes suelen marcarse como una característica del activismo judicial Cross \& LINDQUIST, 2007, p. 176.

${ }^{27}$ Pulido, 2018a, pp. 65-94.

${ }^{28}$ Pulido, 2018b, pp. 293-324. 


\section{b. Las competencias para la creación y aplicación de precedentes judiciales}

La segunda función de la RP es establecer las competencias para la creación y aplicación de los PJ. La RP, en estos términos, es una regla de competencia. Estas reglas se caracterizan por ser reglas secundarias que definen las condiciones para producir actos válidos. Una norma de competencia es aquella que atribuye a la autoridad A el poder normativo de realizar el acto $\mathrm{N}$, mediante el procedimiento (o formas) $\mathrm{P}$, en relación con la materia $\mathrm{M}^{29}$.

Veamos un sencillo ejemplo: una Constitución establece que corresponde al tribunal constitucional, en el marco de un proceso de constitucionalidad abstracta, definir la constitucionalidad por vicios de forma o contenido de los decretos legislativos que dicte el gobierno en los estados de excepción. Esta norma de competencia contempla los siguientes elementos: A (el tribunal constitucional), N (declaración de constitucionalidad o inconstitucionalidad), $\mathrm{P}$ (proceso de constitucionalidad abstracta) y $\mathrm{M}$ (vicios constitucionales de forma y contenido de los decretos legislativos). La validez de un acto de declarar la constitucionalidad o inconstitucionalidad de una norma depende del cumplimiento de las condiciones definidas en la norma de competencia que posibilita el ejercicio de ese acto, esto es, la regla que resulta de la conjunción de las condiciones A, P y M. Así, por ejemplo, si el tribunal constitucional decide acerca de los decretos legislativos por cuestiones distintas a los vicios constitucionales de forma o procedimiento, entonces la decisión es nula o inválida. Lo mismo que si una autoridad distinta a la Corte Constitucional pretende declarar la constitucionalidad de esos decretos, lo cierto es que por más de que se cumplan los requisitos $\mathrm{N}, \mathrm{P}$ y $\mathrm{M}$ el acto es inválido.

En tanto la RP define acerca de competencias se constituye en condición de la validez de determinados actos. En específico, la RP contiene dos tipos de normas de competencia (que, por tanto, condiciona la validez de dos clases de actos normativos) ${ }^{30}$. En primer lugar, la RP es una regla que establece las condiciones para la creación, modificación y derogación de los PJ (una regla de cambio) ${ }^{31}$. Y en segundo lugar la RP es una regla que define las condiciones de la validez para la aplicación de los PJ (una regla de adjudicación ${ }^{32}$ ).

${ }^{29}$ Ferrer-Beltrán, 2000, pp. 147-68. Bulygin, 1992, pp. 201-216. Existen críticas a la tesis según si las reglas de competencia son reglas determinativas o constitutivas. Véase SPAAK, 2003, pp. 89-104 y Arriagada Cáceres, 2017, pp. 93-121. En todo caso, como lo explica Arriagada Cáceres, la noción fundamental de "competencia" es la posibilidad de producir, modificar o derogar normas válidas en las condiciones definidas por las normas de competencia.

${ }^{30}$ Como se expuso en la nota 6 al pie de este trabajo, la RP es el resultado de la síntesis de un conjunto de normas de segundo orden, dentro de las cuales se encuentran normas de competencia. Por esta razón, la RP no es solo una norma de competencia sino una norma que contiene normas de competencia.

${ }^{31}$ HART, 1994, pp. 95-97. En el common law esa dimensión de la RP establece las condiciones para realizar el overruling, esto es, modificar o derogar PJ. Duxbury, 2008, pp. 116-128. Summers \& ENG, 1997, pp. 519-529.

32 HART, 1994, pp. 97-99. 
Como regla de cambio, la RP define 1) las autoridades competentes para crear PJ o introducir cambios en ellos, 2) la condiciones y formalidades (o procedimientos) para hacerlo, y 3) el ámbito o materias en donde se pueden crear precedentes. En relación con las autoridades judiciales competentes para crear, modificar o derogar PJ, diferentes ordenamientos jurídicos establecen que los cambios de PJ solo los puede introducir por la autoridad que creó el PJ o por una autoridad superior ${ }^{33}$. Con base en lo anterior se diferencia entre precedente vertical, precedente horizontal y autoprecedente. El primero es aquel PJ que proviene de sentencias de un juez o tribunal de jerarquía superior. El horizontal es el PJ que proviene de sentencias de jueces o tribunales de la misma jerarquía. Por último, el autoprecedente es el originado en sentencias del mismo juez o tribunal. En diferentes ordenamientos jurídicos se define que los cambios a los PJ solo pueden realizarse sobre el horizontal y el autoprecedente ${ }^{34}$.

$A l$ respecto, la pregunta que surge es ¿tiene sentido afirmar que una autoridad judicial A está vinculada al precedente B si, en todo caso, puede cambiar B? La respuesta es que la RP vinculante depende de que se puedan diferenciar las condiciones de cambio de las condiciones de seguimiento. En tal caso, la RP debe diferenciar dos tipos de contendidos normativos. El primero se relaciona con la forma en que el seguimiento de PJ condiciona la validez de los actos de la misma autoridad que lo crea y el segundo con las condiciones que caracterizan competencia para modificarlos. En la mayoría de organizaciones jurisdiccionales se establece una estructura piramidal con órganos judiciales de cierre y de instancia. En estos contextos, la RP puede establecer que los PJ de los órganos de cierre vinculen verticalmente a las decisiones de los órganos de instancia y pueden, incluso, generar vinculación con sus propias decisiones (autoprecedente). En el derecho constitucional estadounidense, por ejemplo, los PJ de la Suprema Corte vinculan a los tribunales inferiores y los cambios en esos PJ solo pueden ser introducidos por la propia Suprema Corte. Además, existen unas causales a las que debe recurrir la Suprema Corte para justificar el cambio en sus PJ: modificaciones en las fuentes jurídicas; cuando se muestre que el precedente afecta la coherencia o consistencia del derecho, y cuando se muestre que el PJ es inconsistente con el sentido contemporáneo de justicia ${ }^{35}$.

Además, la RP como regla de adjudicación define las condiciones de la validez para la aplicación de los PJ. En este sentido la RP es vinculante en tanto sujeta la validez de

33 Gerhardt, 2008, pp. 9-46; Cross \& Harris, 2012, pp. 26; Pulido, 2018a, pp. 60-63. y Garay, 2013, pp. 224-230

${ }^{34}$ Cross \& Harris, 2012; 159-161 y Magaloni, 2001, p. 38. Es cierto que en las tradiciones jurídicas continentales no suele hablarse de jerarquía judicial. Sin embargo, para efectos de la configuración de la RP, se entiende que un tribunal es superior a otro cuando tiene la competencia de anular sus decisiones mediante algún tipo de recurso ordinario ( $v . g r$ la apelación) o extraordinario $(v \cdot g r$ la casación). De esta materia véase Dobins, 2010, pp. 1460-1466 y Pulido, 2018a, p. 32. Una cuestión que le corresponde responder a la RP es si los PJ pueden cambiarse por autoridades no judiciales, en especial, los Parlamentos. En efecto, en algunos ordenamientos jurídicos las leyes pueden modificar o derogar los PJ ( $v$ gr. el derecho estadounidense); en otros se le excluye esa posibilidad a los Parlamentos ( $v, g r$. el derecho constitucional colombiano).

${ }^{35}$ Shapiro, 2008, pp. 929-956. 
determinados actos normativos a la aplicación de los $\mathrm{PJ}^{36}$. En otras palabras, los actos de una autoridad A están vinculados a un PJ si es el caso que la validez de los actos de A depende de la aplicación del PJ. En este orden de ideas, la RP establece las condiciones en que los sujetos vinculados deben aplicar los PJ. En esta tarea, la RP puede definir las condiciones en que los sujetos vinculados pueden apartarse de un PJ, i.e. las condiciones en que esos sujetos pueden dejar de aplicar un PJ sin afectar la validez de sus actos. Existe, por tanto, una diferencia entre apartarse de un PJ (no aplicarlo a un caso sin que afecte la validez de la decisión) y cambiarlo (derogarlo parcial o totalmente). En el contexto de la RP vinculante, la posibilidad de cambiar los PJ suele limitarse a la modificación del autoprecedente o a los PJ de órganos judiciales inferiores. En cambio, no es extraño que la RP admita que incluso los órganos judiciales inferiores se aparten de los PJ de los órganos judiciales superiores.

Suelen identificarse dos argumentos principales para justificar que un operador judicial se aparte de un PJ: argumentos basados en distinciones ${ }^{37}$ y la pérdida de fuerza de los $\mathrm{PJ}^{38}$. Las distinciones pueden ser de dos tipos ${ }^{39}$ : por un lado, los operadores pueden distinguir entre los hechos que deben resolver en la litis y los que están regulados en el PJ. Esta potestad consiste simplemente en decidir que los hechos a resolver en el litigio no hacen parte de los supuestos de hecho definidos en el PJ. Imaginemos que un PJ exige que si se dan los supuestos a, b y c entones B debe realizar la conducta $\Phi$. Ahora supongamos que el juez se debe pronunciar respecto de los hechos p, q y r. En este caso, desde luego, el juez no está vinculado al PJ en tanto p, q y r no hacen parte del supuesto de hecho de aquel. Lo que suele ocurrir es otra cosa: se argumenta que p, q o $\mathrm{r}$ son suficientemente parecidos a los hechos a, b y c como para justificar ampliar el PJ. En estas circunstancias, no se está pidiendo que el juez aplique el PJ al caso informado por los hechos p, q y r sino que lo extienda a unos hechos distintos (típicamente porque en esos hechos no hay calificación jurídica, esto es, porque hay una laguna). En otras palabras, se está pidiendo al juez la extensión, por analogía, del PJ a unos hechos no regulados por el PJ. No obstante, la autorización de recurrir a la analogía para extender precedentes dependerá - desde luego- de las características y propósitos de cada práctica jurídica, de forma tal que no es posible dar una respuesta general del asunto. En otras palabras, esta posibilidad depende de la forma en que contingentemente se implemente la RP en cada ordenamiento jurídico. Asimismo, los operadores jurídicos pueden plantear un segundo tipo de distinción: la potestad de diferenciar entre los contendidos de las providencias judiciales que son vinculantes de los contenidos que no lo son. Como

\footnotetext{
36 Al respecto véase Arriagada, 2021 pp. 361-396

${ }^{37}$ Duxbury, 2008, pp. 113-116.

38 Cross \& Harris, 2012, pp. 157-159.

${ }^{39}$ De estas formas de distinciones véase López, 2006, pp. 213-220 y Bernal Pulido, 2009, pp. 163-165.
} Sin embargo, en el common law la noción distinguish es más restringida y se refiere a la técnica que emplean los jueces para apartarse de un PJ al encontrar una diferencia fáctica relevante entre el caso precedente (i.e. el caso resuelto por la sentencia que constituye el precedente) y el caso que debe resolver el juez (GARDNER, 2007, p. 71). 
se dijo antes, la RP contempla las condiciones para determinar qué es lo que conforma un PJ. Como consecuencia de la diferenciación entre ratio decidendi y el obiter dictum, los operadores sí están vinculados -entre otros- a la ratio decidendi (y no a la obiter dictum). Obsérvese que en estas circunstancias las distinciones son autorizadas para establecer el precedente vinculante, pero no como argumento para apartarse del mismo.

La pérdida de fuerza de los PJ se refiere a la definición de un conjunto de razones que justifican que un operador judicial pueda apartarse de un PJ. En este caso no es que el operador realice distinciones fácticas (entre los hechos de los casos) o normativas (entre los contenidos considerados ratio decidendi y los que son obiter dicta), sino que -por ejemplo- a pesar de que determinado contenido es ratio decidendi y que no es posible plantear distinciones fácticas, en todo caso el operador judicial puede apartarse del PJ. En efecto, la RP puede contemplar varias razones que tienen la capacidad de superar la fuerza vinculante del PJ, esto es excepciones a la vinculatoriedad del PJ del que se trate. En este orden de ideas, la RP puede establecer: a) una vinculatoriedad estricta, en el que los actos normativos vinculados están sujetos invariablemente al PJ, sin contemplar, por tanto, razones capaces de superarlo; o b) una vinculatoriedad limitada, es decir, que se aceptan excepciones a la sujeción al $\mathrm{PJ}^{40}$.

\section{c. La definición de los criterios para establecer el significado de los precedentes judiciales}

El funcionamiento de la RP depende de que sea posible comprender el significado de los textos de las providencias judiciales que constituyen a los PJ. Los PJ, como se ha dicho, son normas generales y abstractas formuladas en providencias judiciales. Se supone, por tanto, que el lenguaje empleado en esas providencias debe ser capaz de comunicar el PJ. Para comprender esta idea, resulta pertinente recurrir a la diferencia entre significado literal (o semántico) y significado pragmático o contextual ${ }^{41}$.

El significado literal está en función de los símbolos lingüísticos empleados en los textos jurídicos; la referencia o la clase de objetos a los que se refiere el lenguaje; y el sentido (o contenido) del lenguaje, a saber, el conjunto de criterios que deben acreditarse para establecer que cierto objeto (o conjunto de objetos) forman parte de la clase de objetos referenciados por el término ${ }^{42}$. El significado literal de las disposiciones jurídicas en general, y de las providencias judiciales que formulan los PJ en particular, está dado por las relaciones de significación (en función de la referencia y el sentido de los términos lingüísticos) de las expresiones empleadas en ellas.

Volvamos al ejemplo del PJ mencionado antes: un adulto mayor demanda para que las empresas prestadoras de salud le suministren pañales, por cuanto sufre una enfermedad

40 PeCZenik, 1997, pp. 463.

${ }^{41}$ Speaks, 2014. Es oportuno aclarar que no se está intentando proponer una descripción exhaustiva de las técnicas de interpretación de PJ. Lo que se intenta mostrar es que existen unos presupuestos lingüísticos básicos para la formulación de normas e general y de PJ en particular. De la materia véase MARMOR, 2011, pp. 136-159.

${ }^{42}$ Flanagan, 2010, pp. 255-271 
que le ha provocado incontinencia urinaria. Para decidir se discutieron los argumentos a favor y en contra de la petición. Piénsese que se consideraron los argumentos económicos que desaconsejan otorgar ese derecho (razón $p$ ); se consideró, además, que la falta de atención a la incontinencia afecta la salud de los adultos mayores (razón q); y que cuando los adultos mayores no tienen suficientes recursos económicos, el Estado debe asistirlos (razón $s$ ). Se decidió a favor del solicitante y se definió: "A los adultos mayores, las entidades prestadoras de salud deberán proveerlos de pañales en aquellas situaciones en que por su situación médica se hagan exigibles".

En este PJ, el conjunto de propiedades que caracterizan la acción prescrita está determinada, entre otros, por el significado del término "pañales". La semántica de esa disposición depende, por tanto, de la referencia y el sentido de este término. La referencia de "pañales" corresponde, entonces, al conjunto de objetos que podemos calificar como tales. El sentido, por su parte, corresponde al conjunto de criterios que empleamos para incluir o excluir los objetos de la categoría "pañales". Así, los individuos (en tanto hablantes competentes) pueden, en la mayoría de los casos, comprender el tipo de objetos que las entidades deben entregar en virtud del precedente del ejemplo. Esto es así, toda vez que conocen (y comparten) el conjunto de reglas y convenciones que les permite formar el contenido significativo de la expresión "pañales”, y mediante este, determinar los objetos designados.

Algunos piensan que una característica necesaria de las inteligibilidad de las reglas es que el significado de sus formulaciones se independice por completo (se "atrinchere") de sus razones subyacentes (es decir, que el funcionamiento de la regla depende de que el PJ se independice de $p, q$ y $s)^{43}$. No obstante, no es cierto que para que la regla sea comprensible (en tanto regla) sea necesario ese atrincheramiento. Lo que resulta fundamental para la inteligibilidad de la regla es que los individuos puedan comprender el significado del lenguaje empleado por las autoridades que formulan la regla, y que lo hagan sin que sea necesario que recurran a un nuevo razonamiento evaluativo acerca de las razones que las justifican.

Pero el significado de las palabras y las oraciones no se reduce a las funciones semánticas (y sintácticas) del lenguaje, pues depende también de las consideraciones pragmáticas dadas por el contexto de aplicación y el contexto normativo ${ }^{44}$. El contexto de aplicación se refiere al conjunto de hechos que son conjuntamente conocidos (presupuestos) por los individuos, y gracias a ello el significado es comprendido por los participantes de la práctica lingüística. Así, por ejemplo, presuponemos que si pedimos un filete de carne en un restaurante, el mesero nos traerá un filete cocido. Si nos traen un trozo crudo de carne, le diremos al mesero que no "entendió" nuestra orden aun cuando semánticamente este trozo sea un filete.

El contexto normativo, por otro lado, se refiere a las razones que justifican la práctica lingüista en la que estamos involucrados. En el derecho hay buenos ejemplos de cómo

\footnotetext{
43 SChauer, 2004, pp. 113-136

${ }^{44}$ Korta \& Perry 2015; Marmor, 2011, 136-145.
} 
el contexto normativo afecta la comprensión del lenguaje. En el derecho codificado (y en especial la regulación de los títulos valores) los participantes entienden que existen razones ( $v . g r$ la seguridad en las transacciones comerciales) para darle una prevalencia (o autonomía) semántica del lenguaje empleado; por ejemplo, en una letra de cambio. Asimismo, existen diferentes prácticas jurídicas que admiten la relevancia de un conjunto delimitado de razones subyacentes para comprender el significado de los $\mathrm{PJ}^{45}$.

La cuestión de la relevancia de las razones subyacentes está en función de si la RP reconoce (o no reconoce) a los operadores jurídicos la potestad de adaptar el contenido semántico (o significado literal) de los PJ a la luz del contexto en que los PJ fueron definidos. Esto es, en otras palabras, si los operadores jurídicos tienen la potestad de ampliar o reducir el contenido semántico de los PJ en función de las razones subyacentes que justificaron la creación del PJ. Aunque esta potestad implica, en alguna medida, que se supere la "semántica" de las disposiciones jurídicas que constituyen los PJ, ello no significa, de forma necesaria, que se modifique el significado de ese PJ (esta potestad no implica necesariamente modificar o cambiar el PJ). El contenido de la norma se reduce cuando se incluye una propiedad que no hace parte de las condiciones semánticas de los términos empleados en el precedente. Además, se amplía cuando se excluye una propiedad que hace parte del significado literal de los términos empleados en el precedente.

Volvamos al ejemplo: luego de evaluar las razones $p$ (argumentos económicos de sostenibilidad del sistema de salud que desaconsejan otorgar el derecho), q (daño en la salud de los adultos mayores) y s (los recursos económicos de los adultos mayores) se definió que "las empresas prestadoras de salud deberán proveer a los adultos mayores de pañales en aquellas situaciones en que por su situación médica se hagan exigibles”. Esto quiere decir que, de acuerdo con el contenido exclusivamente semántico del PJ, se pueden exigir pañales (propiedad $a$ ) siempre que se acredite que el beneficiario es un adulto mayor (propiedad $b$ ) que carece de recursos suficientes para solventar el pago de los pañales (propiedad c), y que estos son exigidos por su situación médica (propiedad d). Imaginemos un caso en el que un adulto mayor solicita que le sean suministrados pañales. La entidad responsable responde que ya le fueron suministrados pañales de tela y que, por tanto, la solicitud es improcedente. La decisión de considerar que los pañales de tela dentro del ámbito del PJ resultan irrazonables en tanto justamente del contexto de la formulación del PJ se deriva claramente que el propósito es mejorar la calidad de vida de los adultos mayores. En otras palabras, aun cuando un pañal de tela, en un sentido estrictamente semántico o literal, cumple con la exigencia definida en la propiedad $a$; lo cierto es que una aplicación del contexto normativo nos lleva a concluir que un pañal de tela es contradictorio con la razón $q$, por lo que una decisión en tal sentido (es decir, considerar que la entidad cumplió con la obligación) sería contraria

${ }^{45}$ Las razones subyacentes son aquellas consideraciones que justifican la adopción de una regla jurídica (por ejemplo, las razones $q$ y $s$ del caso empleado en párrafos anteriores). 
a la función excluyente del $\mathrm{PJ}^{46}$. En estos casos, podríamos decir que se hace necesario aplicar la atribución de reducir el significado literal del PJ restringiendo la propiedad $p$ a la propiedad $p-1$.

Posteriormente, otro adulto mayor reclama, además de pañales, crema antipañalitis y paños húmedos. La empresa de salud autoriza los pañales, pero rechaza la solicitud de la crema y los paños. Por su parte, el juez que decide amplía el alcance semántico de la regla e incluye tanto la crema como los paños al quedar demostrado que el uso de pañales en personas adultas exige el uso de esos elementos para evitar daños en la salud (pañalitis e infecciones). En efecto, aun cuando estos productos no hacen parte de la designación del término "pañales", el juez decidió incluirlos, como consecuencia de las razones $q$ y s. Este caso es un ejemplo de ampliación del alcance semántico del precedente, pero -en cualquier caso- no representa una reevaluación de las razones que conforman el contexto normativo del PJ.

El propósito de estos ejemplos es mostrar la relación entre los PJ y las razones subyacentes. En algunas prácticas normativas se espera que las disposiciones jurídicas funcionen con exclusión de las razones subyacentes (este puede ser el caso de lo que, en derecho comercial, se conoce como "autonomía de los títulos valores”). En otras prácticas, por el contrario, las reglas no solo no excluyen a determinadas razones subyacentes, sino que de hecho las incluyen como criterio relevante para determinar su significado (este es el caso de los criterios que suelen incluirse para determinar la ratio decidendi en el common law ${ }^{47}$ ). Es posible, por tanto, que la RP incluya, como consideración normativa relevante para establecer el significado del PJ, a las razones que llevaron al juez a crear el PJ (o sea, las razones determinantes del contenido del PJ). Pero, asimismo, la potestad de adaptar el contenido semántico del PJ no autoriza al juez a recurrir a cualquier tipo de consideración. Si se espera que los PJ funcionen como reglas, por tanto, el juez no puede recurrir a razones que resulten contrarias al significado del precedente, y, además, no puede reabrir el balance de las razones que fueron evaluadas en el PJ. De hecho, lo que permite dicha potestad es consultar esas razones para comprender, de manera adecuada, el contexto del PJ. En suma, la identificación de, por ejemplo, la ratio decidendi involucra la definición del porqué de la regla respectiva. Pero identificar las razones que constituyen ese "porqué" no implica reevaluarlo: el entendimiento de las palabras que se emplean en un PJ depende de la comprensión de esas razones, no de su reevaluación ${ }^{48}$.

${ }^{46}$ Por el significado literal es verdad que un pañal usado es un pañal. Pero nadie estaría dispuesto a aceptar que suministrar un pañal usado es un acto satisfactorio de la obligación establecida en el precedente. En otras palabras, aun cuando se pueda afirmar que se está haciendo una distinción (distinguish) para ajustar el contenido semántico del PJ, esto no significa que se esté modificando el PJ ni que se esté resolviendo un conflicto entre dos normas.

${ }^{47}$ Duxbury, 2008, pp. 58-110.

${ }^{4}$ Desde luego que si la RP establece un conjunto de razones como consideración normativa relevante para establecer el significado del PJ, entonces esas razones forman parte del PJ. La actividad del operador jurídico para comprender esas razones, y por tanto para establecer el contenido del PJ, no implica que necesariamente se esté reevaluando el PJ. Véase Pulido, 2018a, pp. 322-323. De la distinción entre evaluar un contenido y comprenderlo véase ENDICOTT, 2014, pp. 46-56. 
De acuerdo con lo dicho, es posible remarcar tres puntos. Primero, son diferentes las potestades de adaptar el contenido semántico del PJ y la de modificarlo (y de lo primero no se sigue lo segundo). Puede darse que un juez ajuste el contenido semántico de un PJ, pero que no tenga la autoridad para modificarlo. Segundo, cuando se ejerce la potestad de adaptar el contenido semántico, no se modifican las razones subyacentes del PJ (i.e. las razones que en específico hacen parte de la regla que constituye el PJ), ni sus relaciones con las razones contrarias a la conducta exigida. Por tanto, la potestad de adaptar el contenido semántico implica autorizar la consulta de las razones subyacentes (por ejemplo, las razones $q$ y $s$ ), pero no a modificar la relación de estas con las razones excluidas contrarias a la conducta exigida (por ejemplo, la razón $p$ ). Tercero, la definición del contenido de esas potestades depende de la configuración de la RP en cada ordenamiento jurídico.

\section{LA REGLA DE PRECEDENTE COMO OBLIGACIÓN}

Existe una diferencia entre vincular y obligar. Una RP es vinculante en el sentido que se constituye una condición para la validez de los actos de los operadores jurídicos. La consecuencia de desconocer la RP es la invalidez o nulidad del acto respetivo. La RP puede - de forma contingente- prescribir la aplicación de PJ (la RP como obligación) ${ }^{49}$. Asimismo, una RP es obligatoria cuando sobre la aplicación de PJ. En este caso, la RP no pretende definir las condiciones de validez de un acto sino exigir una conducta: aplicar PJ.

La existencia de la RP como obligación no es una consecuencia del carácter vinculante de la RP. Con otros términos, no es cierto que las normas que vinculen también obliguen (o prescriban). En realidad, es conceptualmente compatible que un acto sea válido pero prohibido (por ejemplo la venta de una cosa ajena hurtada). Lo que ocurre es que generalmente los operadores jurídicos no solo están vinculados al derecho vigente, sino que además tienen la obligación de aplicarlo. En otras palabras, el derecho vigente (dentro del que, en virtud de la RP, se encuentran los PJ) se constituye en una condición de validez de los actos jurídicos ( $v$ gr r sentencias judiciales), pero suele existir también el deber de aplicarlo. Es por esta razón que los teóricos del derecho han observado que los ordenamientos jurídicos complementan las reglas de competencia (que generan potestades) con reglas prescriptivas acerca de cómo emplear esas potestades (pueden existir normas que prescriban acerca de la forma de usar competencias). De acuerdo con

${ }^{49}$ La distinción entre vinculación y obligación en el marco de la regla de precedente ha sido recientemente estudiada en Arriagada, 2021 pp. 361-396 y NúÑez Vaquero, 2021, pp. 329-360. Concretamente, este trabajo sigue la idea de estos autores cuando sostienen que es conceptualmente distinto afirmar que los PJ son obligatorios a afirmar que son vinculantes. No obstante, en este apartado del trabajo se subraya que un ordenamiento jurídico puede contingentemente establecer contenidos prescriptivos en la regla de precedente. Como se dijo antes, la regla de precedente es en realidad una regla compleja que puede involucrar la idea de regla secundaria en dos sentidos. Por un lado, una función constitutiva de las formas válidas de crear, identificar, aplicar PJ. Por el otro, una metanorma (o reglas sobre reglas) en la que la regla de precedente -contingentemente- prescribe la aplicación de PJ. 
esto, es posible, aunque no necesario, que los ordenamientos jurídicos obliguen a los operadores jurídicos a usar la RP en el ejercicio de sus competencias.

Esta diferencia se comprende mejor al analizar las consecuencias del desconocimiento de cada una de esas dimensiones de la RP. El desconocimiento de las RP vinculante genera la invalidez y eventual nulidad de los actos jurídicos. En tanto la RP es una condición necesaria de la validez de los actos entonces su desconocimiento implica la invalidez de los mismos ${ }^{50}$. En estos términos, la invalidez es la consecuencia necesaria de no observar las condiciones establecidas en las RP vinculantes ${ }^{51}$. Por el contrario, no existen consecuencias necesarias para el incumplimiento de las reglas prescriptivas en general, ni de la RP como obligación en particular. Lo que ocurre es que los ordenamientos jurídicos eventualmente contemplan sanciones como respuesta al desobedecimiento de sus prescripciones. No obstante, la existencia de sanciones es independiente de la existencia de los deberes jurídicos creados por las reglas ${ }^{52}$. Las sanciones son las consecuencias contingentes del incumplimiento de las reglas que obligan a seguir la RP. La nulidad (o invalidez) es la consecuencia necesaria del incumplimiento de la RP vinculante. En suma, es conceptualmente posible una RP vinculante sin sanciones y conceptualmente imposible una RP vinculante sin nulidades ${ }^{53}$.

En estricto sentido, no se pueden contemplar sanciones como consecuencia del no seguimiento a la RP vinculante. Esta regla es una norma compleja que define las condiciones en que un acto jurídico puede ser creado, modificado, derogado o aplicado. Es decir, la consecuencia jurídica ante el incumplimiento de RP es la invalidez del acto. Lo que puede ocurrir es que se contemplen deberes cuyo contenido incluya la realización de un acto jurídico y, por tanto, el seguimiento de reglas de competencia. Una decisión judicial, por ejemplo, es un acto jurídico cuya validez depende del cumplimiento de las condiciones establecidas en reglas de competencia (incluida, de ser el caso, la RP vinculante). Si los jueces no cumplen con estas condiciones sus sentencias serán inválidas. Además de ello, suelen existir reglas que prescriben que los jueces resuelvan, mediante sentencias, los casos sometidos a su competencia. En definitiva, y en relación con las decisiones judiciales, es posible diferenciar entre a) las reglas de competencia que definen sus condiciones de validez y b) las reglas que obligan a proferir sentencias (o incluso que obligan a aplicar la RP).

\footnotetext{
${ }^{50}$ Pulido, 2018a, pp. 325-381.

${ }^{51}$ HART. 1994, pp. 33-38.

52 Raz, 1990, pp. 154-162.

53 Acerca del carácter contingente de las sanciones en la configuración de la regla de precedente véase Pulido, 2018a, pp. 325-381 y NúÑez Vaquero 2021, p. 337-338.
} 


\section{BIBLIOGRAFÍA}

Alchourrón, Carlos y Bulygin, Eugenio, 1987: Introducción a la metodología de las ciencias jurídicas $y$ sociales, Buenos Aires: Astrea.

Alexander, Larry \& Sherwin, Emily, 2007: “Judges as Rulemakers”, en Douglas Edlin (editor), Common Law Theory, Cambridge: Cambridge University Press, pp 27-50.

Arriagada Cáceres, María Beatriz, 2017: "Normas de competencia y normas acerca de la competencia. Eludiendo las reglas constitutivas". Doxa. Cuadernos de Filosofía del Derecho, volumen 40, pp. 93-121, Disponible en: https://doxa.ua.es/article/ view/2017-n40-normas-de-competencia-y-normas-acerca-de-la-competencia.

Arriagada Cáceres, María Beatriz, 2021: "Las dos caras del precedente vinculante", en A. Núñez Vaquero, M. Beatriz Arriagada Cáceres y I. Hunter Ampuero, Teoría y práctica del Precedente, Valencia: Tirant lo Blanch, pp. 361-396.

Cross, Rupert y Harris, J.W., 2012: El precedente en el derecho inglés, Madrid: Marcial Pons.

Bernal Pulido, Carlos, 2009: El neoconstitucionalismo y la normatividad del derecho. Escritos de derecho constitucional y filosofía del derecho, Bogotá: Universidad Externado de Colombia.

Bronaugh, Richard, 1987: Persuasive Precedent, en L. Goldstein (editor), Precedent in Law, Oxford: Clarendon Press, pp. 216-247.

Bravo-Hurtado, Pablo, 2013: "hacia los precedentes en chile: reforma procesal civil y fuentes del derecho", Revista chilena de derecho, volumen 40, número 2, pp. 549-576. https://dx.doi. org/10.4067/S0718-34372013000200008

Bulygin, Eugenio, 1992: "On norms of competence", Law and Philosophy, volume 11, pp. 201-216. https://doi.org/10.1007/BF01000642.

Cross, Frank \& Lindquist, Stefanie, 2007: The scientific study of judicial activism, Minnesota Law Review, volume 91, número 6, pp. 1752-1784. Disponible en https://www.minnesotalawreview.org/wp-content/uploads/2012/01/CrossandLindquist_Final.pdf [Fecha de consulta: 1.3 .2020$]$.

Coleman, Jules, 2011: The practice of Principle: In Defense of a Pragmatist Approach to Legal Theory, Oxford: Oxford University Press.

Díaz Revorio, Francisco J., 2000: "Interpretación constitucional y sentencias interpretativas", Repertorio Aranzadi del Tribunal Constitucional, número 1, pp. 1799-1824.

Dobbins, Jeffrey, 2010: "Structure and Precedent", Michigan Law Review, volumen 108, número 8, pp. 1453-1496. Disponible en https://repository.law.umich.edu/mlr/vol108/iss8/1/

Duxbury, Neil, 2008: The Nature and Authority of Precedent, New York: Cambridge University Press.

Flanagan, Brian, 2010: "Revisiting the Contribution of Literal Meaning to Legal Meaning", Oxford Journal of Legal Studies, volumen 30, numero 2, pp. 255-271, https://doi.org/10.1093/ ojls/gqp030

Ferrer-Beltrán, Jordi, 2000: Las normas de competencia un aspecto de la dinámica jurídica, Madrid: Centro de Estudios Políticos y Constitucionales.

Garay, Alberto, 2013: La doctrina del precedente en la Corte Suprema, Buenos Aires: Abeledo Perrot.

Gardner, John, 2007: "Some Types of Law", en Douglas Edlin (editor), Common Law Theory, Cambridge: Cambridge University Press, pp. 51-77.

Gerhardt, Michael J., 2008: The Power of Precedent, Oxford: Oxford University Press.

Guastini, Riccardo, 1999: Distinguiendo, Barcelona: Gedisa.

Guastini, Riccardo, 2001: Estudios de teoría constitucional, México: Fontamara.

Hart, H. L. A., 1994: The concept of law (2a. Ed.) P. Bulloch y J. Raz (editores), Oxford: Clarendon Press.

Hernández Marín, Rafael, 2002: Introducción a la teoría de la norma jurídica (2a Ed.), MadridBarcelona, 2002. 
Hernández Marín, Rafael, 2008: Las obligaciones básicas de los jueces, Madrid: Marcial Pons.

KomareK, Jan, 2013: "Reasoning with previous decisions: beyond the doctrine of precedent", American Journal of Comparative Law, volumen 61, número 1, pp. 149-171. Disponible en www.jstor.org/stable/41721717 [Fecha de consulta: 1.3.2020]

KorTa, Kepa \& Perry, John, 2015: "Pragmatics”, En E. Zalta (editor) The Stanford Encyclopedia of Philosophy. Disponible en http://plato.stanford.edu/entries/pragmatics/\#Aca [Fecha de consulta: 1.3.2020].

LóPEz, Diego, 2006: El derecho de los Jueces. Bogotá: Legis.

Marmor, Andrei, 2011: Philosophy of Law, Princeton: Princeton University Press.

Marshall, Geoffrey, 1997: "What is binding in a precedent", En: N. Mac Comrmick y R. Summers (editors), Intepreting Precedents. Aldershot: Ashgate/Dartmouth, pp. 503-517.

Moreno Cruz, Pablo, 2017: "efectos inter comunis: una acción de tutela colectiva y obligatoria", en Ramiro Bejarano Guzmán, Pablo Moreno Cruz, Marcela Rodríguez Mejía (editores), Aspectos procesales de la acción de tutela, Bogotá: Universidad Externado de Colombia, p. 61-119.

Moreso, José Juan, \& Queralt, Jahel, 2014: Bosquejo de Dworkin: la imbricación entre el derecho y la moralidad, Isonomía (México), volumen 41, pp. 143-174. Disponible http://www. scielo.org.mx/scielo.php?script =sci_arttext\&pid =S1405-02182014000200007\&lng =es\& tlng=es . [Fecha de consulta: 1.3.2020]

NúÑez Vaquero, Álvaro, 2016: "Sin precedentes: una mirada escéptica a la regla del stare decisis”, Doxa. Cuadernos de Filosofía del Derecho, número 39, pp. 127-156. https://doi. org/10.14198/DOXA2016.39.08

NúÑEz Vaquero, Álvaro, 2021: “¿Son obligatorios los precedentes? La regla del precedente como norma(s) constitutiva(s)”, en A. Núñez Vaquero, M. Beatriz Arriagada Cáceres y I. Hunter Ampuero, Teoría y práctica del Precedente, Valencia: Tirant lo Blanch, pp. 329-360.

Peczenik, Aleksander, 1997: "The Binding force of precedent", en: N. Mac Comrmick y R. Summers (editors), Intepreting Precedents. Aldershot: Ashgate/Dartmouth, pp. 461-479.

Pulido-Ortiz, Fabio, 2018a: Jueces y reglas. La autoridad del precedente judicial. Bogotá: Universidad de La Sabana.

Pulido-Ortiz, Fabio Enrique, 2018b: "Poderes normativos de la Corte Constitucional colombiana”, Ius et Praxis (Talca), volumen 24, número 3, pp. 309-334, Disponible en https:// scielo.conicyt.cl/scielo.php?script =sci_arttext\&pid $=$ S0718-00122018000300309\&lng $=e$ s\&nrm = iso [Fecha de consulta: 1.3.2020]

RAZ, Joseph, 1979: The authority of law: Essays on Law and Morality, Oxford: Oxford University Press, 1979. Raz, Joseph, 1990: Practical Reason and Norms, Oxford: Oxford University Press.

RAZ, Joseph, 2019: "Normative Powers”, Oxford Legal Studies Research Paper, número 36, disponible en : https://ssrn.com/abstract $=3379368$ or http://dx.doi.org/10.2139/ssrn. 3379368 [Fecha de consulta: 1.3.2020]

SHAPIRO, David, 2008: "The role of precedent in constitutional adjudication: an introspection", Texas Law Review, volumen 86, número 5, pp. 929-956.

SCHAUER, Frederick, 2004: Las reglas en juego. Un examen filosófico de la toma de decisiones basada en regles en el derecho y en la vida cotidiana, Barcelona: Marcial Pons.

SCHAUER, Fredrick, 2013: Pensar como un abogado. Una nueva introducción al razonamiento jurídico (T. Schleider, trad.), Madrid: Marcial Pons.

SpAAK, Torben, 2003: "Norms that Confer Competence", Ratio Iris, volumen 16, pp. 89-104.

SPEAKs, Jeff, 2014: "Theories of Meaning”, en: E. Zalta (editor), The Stanford Encyclopedia of Philosophy, https://plato.stanford.edu/archives/win2019/entries/meaning/> [Fecha de consulta: 1.3 .2020$]$

Summers, Robert y Eng, Svein, 1997: “Departures from Precedent”. En: N. Mac Comrmick y R. Summers (editors), Intepreting Precedents. Aldershot: Ashgate/Dartmouth, pp. 519-529. 
Twining, William y Miers, David, 2010: How to do thing with rules, Cambridge: Cambridge University Press.

VON Wright, Georg Henrik, 1963: Norm and Action. A Logical Inquiry, Londres: Routledge \& Kegan Paul.

WALDRON, Jeremy, 2020: "The Rule of Law". En Edward N. Zalta (ed.), The Stanford Encyclopedia of Philosophy, disponible en https://plato.stanford.edularchives/sum2020/entries/rule-of-law/

Zhou, Han-Ru, 2019: "Erga Omnes or Inter Partes? The Legal Effects of Federal Courts Constitutional Judgments", Canadian Bar Review, volume 97, número 2, pp. 275-299. Disponible en https://cbr.cba.org/index.php/cbr/article/view/4540 [Fecha de consulta: $1.3 .2020]$ 